\title{
Dynamic Changes in Plasma MicroRNAs Have Potential Predictive Values in Monitoring Recurrence and Metastasis of Nasopharyngeal Carcinoma
}

\author{
Xia Xu, ${ }^{1}$ Juan Lu, ${ }^{2}$ Fan Wang, ${ }^{2}$ Xiong Liu $\mathbb{D}^{,},{ }^{2}$ Xiaohong Peng, ${ }^{2}$ Bolong Yu, \\ Feipeng Zhao, ${ }^{2}$ and Xiangping $\mathrm{Li} \mathbb{D}^{2}$ \\ ${ }^{1}$ Department of Otolaryngology-Head and Neck Surgery, Guangzhou General Hospital of \\ People's Liberation Army of China, Guangzhou, Guangdong 510010, China \\ ${ }^{2}$ Department of Otolaryngology-Head and Neck Surgery, Nanfang Hospital, Southern Medical University, \\ Guangzhou, Guangdong 510515, China
}

Correspondence should be addressed to Xiangping Li; li321162@qq.com

Received 24 July 2017; Revised 3 October 2017; Accepted 15 October 2017; Published 18 January 2018

Academic Editor: Leon Spicer

Copyright (C) $2018 \mathrm{Xia} \mathrm{Xu}$ et al. This is an open access article distributed under the Creative Commons Attribution License, which permits unrestricted use, distribution, and reproduction in any medium, provided the original work is properly cited.

\begin{abstract}
Although circulating microRNAs (miRNAs) have already proven to be useful as diagnostic and prognostic biomarkers in nasopharyngeal carcinoma (NPC), the potential of these molecules to monitor patients over time has been less explored. This study aimed to analyze dynamic changes in plasma miRNAs before and after treatment and explore their clinical significance in monitoring recurrence and metastasis of NPC. Candidate miRNAs were screened by microarray analysis and validated by real-time quantitative polymerase chain reaction (RT-qPCR). In the follow-up cohort including 102 patients, blood samples (plasma) were collected before the treatment initiation, 3 months, 6 months, and 12 months after treatments, and at the time of any recurrence or metastasis. Among these plasma miRNAs, miR-9-3p, miR-124-3p, miR-892b, and miR-3676-3p were significantly upregulated $(P$ $=0.018, P=0.039, P=0.001$, and $P=0.01$, resp.) after treatment compared with pretreatment, and the four plasma miRNAs were downregulated again at recurrence or metastasis $(P<0.001, P=0.015, P=0.003$, and $P=0.026$, resp.). The dynamic changes in plasma miRNAs after treatment reflect the outcome of the disease and have the potential to monitor recurrence and metastasis in patients with NPC.
\end{abstract}

\section{Introduction}

Nasopharyngeal carcinoma (NPC) is one of the common malignant tumors in Southeast Asia with a high rate of local invasion and locoregional lymphatic metastasis [1]. Currently, the incidence of NPC in Southern China is especially high, at nearly 30 per 100,000 of population [2]. Despite the great advances in intensity-modulated radiotherapy, more than $30 \%$ of patients eventually develop recurrence or distant metastasis. Recurrence or metastasis is still the leading cause of death when curative treatment is no longer possible [3]. The comprehensive assessment of tumor response includes clinical examination, nasendoscopy with or without biopsy, Epstein-Barr virus (EBV) DNA or virus capsid antigenspecific IgA (VCA-IgA) titer measurement, and radiological imaging [4]. EBV is important in the development and progression of NPC. The plasma EBV-DNA level is currently recognized as the prognostic factor for NPC, but fake positive and negative results may be obtained due to the universality of EBV infection [5]. Recent studies have shown that specific genetic changes, such as dysregulation of microRNA expression or DNA methylation, may also predict the diagnosis and prognosis of NPC $[6,7]$. Nevertheless, several issues need to be overcome before clinical genetic testing can become routine, including discrepancies between individual cases and inconsistencies in detection standards. This leads to a pressing need to identify an efficient tool to monitor recurrence or metastasis during the follow-up period of NPC, especially in Southern China.

MicroRNAs (miRNAs) are small noncoding RNAs that play an important regulatory role in a wide range of biological 
and pathological processes, especially in tumorigenesis and progression of various cancers [8]. Circulating miRNAs (plas$\mathrm{ma} /$ serum miRNAs) have been proved to be present in the bloodstream in a remarkably stable form and serve as promising novel minimally invasive biomarkers for diagnosis and prognosis in multiple cancers, such as lymphoma and breast, prostate, ovarian, pancreatic, gastric, colorectal, and lung cancer [9-11]. However, identifying tumor-specific miRNAs is hampered by the fact that blood also contains miRNAs derived from other physiological or pathological activities, whose levels correlate with individual differences [12]. Additionally, the levels of circulating miRNAs generally fluctuate between different patients, and it is difficult to define a normal range of circulating miRNA levels. Therefore, some researchers believe that a paired comparative study between pre- and posttreatment can provide more accurate results to evaluate the therapeutic outcome and monitor tumor recurrence or metastasis $[13,14]$.

Several reports are available about circulating miRNAs in NPC tumorigenesis [15]. Liu et al. reported that the plasma five-miRNA-based biomarker model might provide a novel strategy for NPC diagnosis and prognosis [16]. Moreover, the four-serum miRNA signature might add prognostic value to the TNM staging system and provide information for personalized therapy in NPC [17]. Zhang et al. indicated that circulating EBV miRNAs might constitute new useful serological biomarkers for diagnosing NPC and predicting treatment efficacy [18]. However, the dynamic changes in these dysregulated miRNAs at different time points using self-paired plasma samples during the regular follow-up have never been explored.

In a previous study, the microarray analysis was performed to identify the miRNA expression signature in patients with NPC, and 33 differentially expressed miRNAs were identified. Among these 33 miRNAs, the low level of plasma miR-9 was significantly correlated with worse lymphatic invasion and advanced TNM stage. Interestingly, the plasma miR9 was significantly elevated in 3-month posttreatment plasma compared with that in pretreatment samples [19]. This study aimed to analyze the dynamic changes in these dysregulated miRNAs in self-paired plasma samples after radiochemotherapy and explore their clinical potential as useful noninvasive biomarkers for monitoring recurrence and metastasis in patients with NPC.

\section{Materials and Methods}

2.1. Patient Cohort. A total of 122 patients newly diagnosed with NPC and 45 healthy donors were recruited prospectively from Nanfang Hospital (Southern Medical University, Guangzhou, China) between January 2011 and July 2014. None of them had received radiotherapy or chemotherapy earlier. Before treatment, blood samples taken from 20 patients and 10 healthy donors were used for miRNA microarray analysis, and additional samples from 102 patients and 35 healthy donors were used for validation. Then the 102 patients were followed up to September 2016 after treatment; those who could not return on time were excluded. The healthy donors were carefully selected to match the gender and age distribution of patients with NPC. The clinical classification for NPC was based on the seventh edition of the Union for International Cancer Control (UICC) TNM staging system. All patients were treated with a uniform protocol of imageguided intensity-modulated radiotherapy and/or cisplatinbased concurrent chemotherapy following induction chemotherapy according to the National Comprehensive Cancer Network Guidelines [20]. Informed consent was obtained from all individuals, and the research protocols were approved by the ethics committee of Nanfang Hospital.

After the completion of all treatments, the patients with NPC were followed up for 12-36 months, once every 3 months during the first and second years and every 6 months thereafter. All tumor recurrences and metastases were documented by imaging studies along with pathological verification if the lesions were accessible and the patient agreed. In the long-term follow-up study, the blood samples of patients with NPC were collected at four time points, including before treatment and 3 months, 6 months, and 12 months after treatment. Once tumor recurrence or metastases occurred, the blood samples were collected timely before the salvage therapy was performed.

2.2. Plasma Sample Collection, RNA Isolation, and miRNA Microarray Analysis. Whole blood $(8 \mathrm{~mL})$ was drawn into ethylene diamine tetraacetic acid- (EDTA-) containing tubes, mixed well, and kept at room temperature. Then, blood samples were centrifuged at $1500 \mathrm{~g}$ for $10 \mathrm{~min}$ to separate cellular fractions and plasma. Finally, aliquot plasma was stored at $-80^{\circ} \mathrm{C}$ until further processing. All blood samples were processed and completed within $4 \mathrm{~h}$ after collection.

Total RNA was extracted from plasma specimen with the TRIzol LS reagent (Invitrogen, CA, USA) and the RNeasy Mini kit (Qiagen, CA, USA) according to the manufacturers' instructions. In detail, $500 \mathrm{~mL}$ of plasma was mixed thoroughly with $1.5 \mathrm{~mL}$ of TRIzol LS reagent, incubated for $5 \mathrm{~min}$ at room temperature, and subsequently mixed with $400 \mathrm{~mL}$ of chloroform. The aqueous phase containing RNA was carefully removed, and RNA was precipitated by adding $100 \%$ ethanol. The mixture was applied to an RNeasy Mini spin column and washed several times. RNA was eluted and stored at $-80^{\circ} \mathrm{C}$ until further processing.

Small RNAs extracted from the plasma samples were labeled using the miRCURY Hy3/Hy5 Power labeling kit and hybridized on the miRCURY LNA Array (Version 16.0, Exiqon, Vedbaek, Denmark). The data was analyzed using GeneSpring GX Software (version 9.0, Agilent Technologies, CA, USA). The threshold value for differentially expressed miRNAs was a fold change $>1.5$ with a $P$ value $<0.05$. The candidate miRNAs were further filtered on the basis of expression levels.

2.3. miRNA Quantitative Real-Time Polymerase Chain Reaction. Quantitative reverse transcription-polymerase chain reaction (PCR) was performed for miRNA quantification using All-in-One miRNA First-Strand cDNA Synthesis Kit and All-in-One miRNA qPCR Kit (GeneCopoeia, MD, USA). The probes were purchased from Invitrogen (Invitrogen, CA, USA). The sequences are listed in Table 1. All reactions 
TABLE 1: All detected plasma miRNA and upstream primer sequences.

\begin{tabular}{lll}
\hline miRNA & Sequence $\left(5^{\prime}-3^{\prime}\right)$ & Primer $\left(5^{\prime}-3^{\prime}\right)$ \\
\hline hsa-miR-9-3p & AUAAAGCUAGAUAACCGAAAGU & ATAAAGCTAGATAACCGAAAGT \\
hsa-miR-92a-2-5p & GGGUGGGGAUUUGUUGCAUUAC & GGGTGGGGATTTGTTGCATTAC \\
hsa-miR-124-3p & UAAGGCACGCGGUGAAUGCC & TAAGGCACGCGGTGAATGCC \\
hsa-miR-214-3p & ACAGCAGGCACAGACAGGCAGU \\
hsa-miR-892b & CACUGGCUCCUUUCUGGGUAGA & ACAGCAGGCACAGACAGGCAGT \\
hsa-miR-3135a & UGCCUAGGCUGAGACUGCAGUG & CACTGGCTCCTTTCTGGGTAGA \\
hsa-miR-3676-3p & CCGUGUUUCCCCCACGCUUU & TGCCTAGGCTGAGACTGCAGTG \\
hsa-miR-4257 & CCAGAGGUGGGGACUGAG & CCGTGTTTCCCCCACGCTTT \\
U6 & CGCAAGGAUGACACGCAAAUUCGU & CCAGAGGTGGGGACTGAG \\
hsa-miR-634 & AACCAGCACCCCAACUUUGGAC & CGCAAGGATGACACGCAAATTCGT \\
hsa-miR-1228-3p & UCACACCUGCCUCGCCCCCC & AACCAGCACCCCAACTTTGGAC \\
\hline
\end{tabular}

were run in triplicate on the Stratagene Mx3005p Real-Time qPCR System (Agilent Technologies, CA, USA). The average levels of U6 small nuclear RNA and two miRNAs (miR-634 and miR-1228-3p), which were not differentially expressed in the microRNA microarray, were used as internal control in plasma miRNA analysis. The differential expression level of plasma miRNA was calculated by the following equation:

$$
\begin{aligned}
\Delta \mathrm{Ct}_{\mathrm{miR}} & =\mathrm{Ct}_{\mathrm{miR}}-\frac{\left(\mathrm{Ct}_{\mathrm{miR}-\mathrm{U} 6}+\mathrm{Ct}_{\mathrm{miR}-634}+\mathrm{Ct}_{\mathrm{miR}-1228}\right)}{3} \\
\Delta \Delta \mathrm{Ct} & =\Delta \mathrm{Ct}_{\text {test }}-\Delta \mathrm{Ct}_{\text {control }} .
\end{aligned}
$$

Fold changes were calculated through relative quantification: $2^{-\Delta \Delta \mathrm{Ct}}[21]$.

2.4. Statistical Analysis. SPSS 16.0 software was used for statistical analysis (SPSS, IL, USA). The Mann-Whitney $U$ test was performed to detect differences in characteristics of study participants and identify differentially expressed miRNAs between NPC and control groups. The expression levels of miRNAs before and after treatment were compared using the paired $t$-test. Repeated-measure analysis of variance (ANOVA) was used for plasma miRNA levels before and 3, 6 , and 12 months after treatment, with the least significant difference tests for multiple comparisons. A $P$ value less than 0.05 was considered statistically significant.

\section{Results}

3.1. Patient Characteristics. The characteristics of the study participants are presented in Table 2. No significant difference was found in the distribution of age and gender between the two cohorts. During the follow-up of 102 patients with NPC, 12 cases had recurrence or metastasis at different time points. The time and sites of recurrence or metastasis are listed in Table 3 .

3.2. Selection and Validation of NPC-Associated Plasma miRNAs. Based on microarray analysis, 16 miRNAs were screened by the two-dimensional hierarchical clustering
TABle 2: Patient and disease characteristics.

\begin{tabular}{lccc}
\hline Characteristics & $\begin{array}{c}\text { Follow-up } \\
\text { cohort } \\
(n=102)\end{array}$ & $\begin{array}{c}\text { Healthy control } \\
(n=35)\end{array}$ & $P$ \\
\hline Age (year) & $46.08 \pm 9.76$ & $43.15 \pm 8.42$ & 0.748 \\
Gender, $n(\%)$ & $76(74.5)$ & $26(74.3)$ & 0.965 \\
$\quad$ Male & $26(25.5)$ & $9(25.7)$ & \\
Female & & & \\
T stage, $n(\%)$ & $6(5.9)$ & & \\
T1 & $28(27.4)$ & \\
T2 & $38(37.3)$ & \\
T3 & $30(29.4)$ & \\
T4 & & \\
N stage, $n(\%)$ & $16(15.7)$ & \\
N0 & $32(31.4)$ & \\
N1 & $43(42.1)$ & \\
N2 & $11(10.8)$ & \\
N3 & & \\
M stage, $n(\%)$ & $99(97.1)$ & \\
M0 & $36(2.9)$ & \\
M1 & & \\
UICC stage, $n(\%)$ & & \\
I & & \\
II & & \\
III & & \\
IV & & \\
\hline & & \\
& & \\
\end{tabular}

analysis, which showed significantly altered levels (Figure 1(a)). Among these 16 miRNAs, the levels of eight differentially expressed miRNAs were tested using an independent cohort (including 102 patients with NPC and 35 healthy volunteers) with quantitative reverse transcription- (qRT-) PCR to confirm the dysregulation of candidate miRNAs. The relative level of each miRNA is shown in Figure 1(b). The results demonstrated that five miRNAs (miR-9-3p, miR92a-2-5p, miR-124-3p, miR-892b, and miR-3676-3p) were 
TABLE 3: Characteristics of recurrence or metastasis cases.

\begin{tabular}{|c|c|c|c|c|c|}
\hline ID & Gender & $\begin{array}{c}\text { Age } \\
\text { (year) }\end{array}$ & TNM stage & $\begin{array}{c}\text { Time } \\
\text { (month) }\end{array}$ & $\begin{array}{l}\text { Recurrence or } \\
\text { metastasis sites }\end{array}$ \\
\hline Case 1 & Male & 42 & T3N1M0 & 20 & Recurrence \\
\hline Case 2 & Male & 39 & T3N2M0 & 8 & Lung \\
\hline Case 3 & Male & 29 & T4N3M0 & 12 & Liver \\
\hline Case 4 & Female & 40 & T3N1M0 & 24 & Bone \\
\hline Case 5 & Male & 45 & T1N3M0 & 24 & $\begin{array}{c}\text { Cervical lymph } \\
\text { node }\end{array}$ \\
\hline Case 6 & Male & 30 & T2N3M0 & 6 & Liver \\
\hline Case 7 & Female & 50 & T4N1M0 & 6 & Recurrence \\
\hline Case 8 & Female & 48 & $\mathrm{~T} 2 \mathrm{~N} 3 \mathrm{M} 0$ & 6 & Liver \\
\hline Case 9 & Male & 56 & T3N2M0 & 12 & Liver \\
\hline Case 10 & Male & 58 & T4N2M0 & 9 & Recurrence \\
\hline Case 11 & Male & 33 & T4N3M0 & 30 & Liver \\
\hline Case 12 & Male & 48 & T4N2M0 & 10 & Recurrence \\
\hline
\end{tabular}

downregulated and three miRNAs (miR-214-3p, miR-3135a, and miR-4257) were upregulated in the plasma of patients with NPC.

3.3. Dynamic Changes in Plasma miRNA Levels after Treatment. The 102 patients with NPC were followed up on time. The paired plasma samples of these patients were collected at different time points, including before, 3 months, 6 months, and 12 months after treatment. The 12 patients with recurrence or metastasis were excluded. The dynamic changes in 8 plasma miRNAs of 90 patients were analyzed during the follow-up period, and the 35 healthy donors were used as controls. As shown in Figure 2(a), the levels of 4 plasma miRNAs (miR-9-3p, miR-124-3p, miR-892b, and miR-3676$3 p)$ were elevated at 3-month posttreatment compared with pretreatment. In contrast, the level of miR-3135a was reduced after treatment. The expression of these five differentially expressed plasma miRNAs tended to go back to basic normal levels after treatment compared with that before treatment. However, the expression levels of another three miRNAs (miR-92a-2-5p, miR-214-3p, and miR-4257) showed no significant differences between pre- and posttreatment. The levels of plasma miR-9-3p and miR-124-3p were significantly elevated at 3-month posttreatment, slightly reduced at 6month posttreatment, and then continued to be elevated at 12-month posttreatment (Figures 2(b) and 2(c)). The levels of plasma miR-892b and miR-3676-3p showed a gradual increase during the follow-up (Figures 2(d) and 2(e)), while the level of miR-3135a showed a gradual decrease over 1 year after treatment (Figure 2(f)). In summary, the dynamic alterations of plasma miRNA levels were observed in patients with NPC after radiochemotherapy during the long-term follow-up.

3.4. Different Changes in Plasma miRNAs Levels at the Time of Recurrence or Metastasis. Most recurrences and metastases $(8 / 12,66.7 \%)$ occurred within 1 year. Liver metastasis $(5 / 12$, $41.7 \%)$ and local recurrence $(4 / 12,33.3 \%)$ were more common. The levels of the eight differentially expressed plasma
miRNAs were detected before the salvage treatment. As shown in Figure 3, the levels of four plasma miRNAs (miR9-3p, miR-124-3p, miR-892b, and miR-3676-3p) statistically were elevated at 3-month posttreatment and decreased at recurrence or metastasis compared with pretreatment $(P<$ 0.05). However, the levels of these 4 miRNAs kept the recovery trend to basic normal levels in another 90 cases of the follow-up cohort without recurrence or metastasis (data not shown). These data suggested that the dynamic changes in these four miRNAs had a potential value as biomarkers for monitoring recurrence and metastasis in patients with NPC.

\section{Discussion}

The aim of this study was to identify dynamic changes in differentially expressed plasma miRNAs in the posttreatment of patients with NPC, especially at the time of recurrence or metastasis, and investigate the potential of plasma miRNA as a biomarker for predicting recurrence or metastasis in patients with NPC. Based on systematic microarray analysis and RT-qPCR validation, the significantly declined level of four plasma miRNAs (miR-9-3p, miR-124-3p, miR-892b, and miR-3676-3p) in patients with NPC showed recovery trend to basic normal levels after treatment but declined again at the time of recurrence or metastasis. These results suggested the potential of the miRNAs as biomarkers for monitoring recurrence and metastasis in patients with NPC.

Circulating miRNAs have been identified as potential noninvasive biomarkers for diagnosis and prognosis in multiple cancers [22]. Several studies showed tumor-specific changes in circulating miRNAs in patients with NPC. Zeng et al. found that four serum miRNAs, including miR-17, miR20a, miR-29c, and miR-223, were differentially expressed in the serum of patients with NPC and proposed that these miRNAs might serve as potential biomarkers for NPC diagnosis [15]. Zhang et al. reported that EBV-encoded miRNAs, miR-BART7 and miR-BART13, might constitute useful new prognostic serological biomarkers for predicting treatment efficacy of NPC [18]. Another study found that differentially 


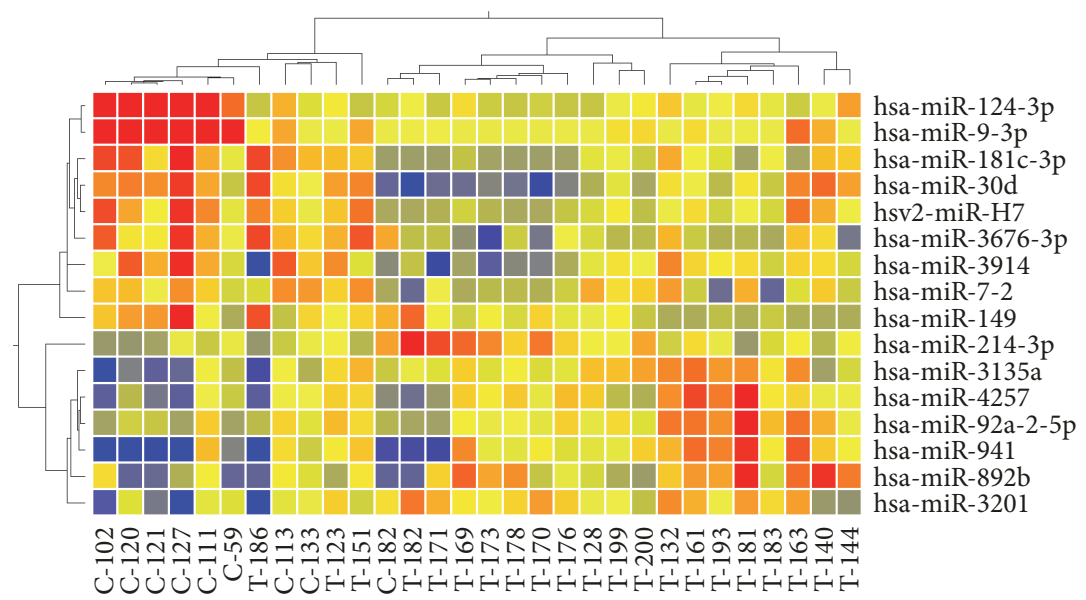

(a)

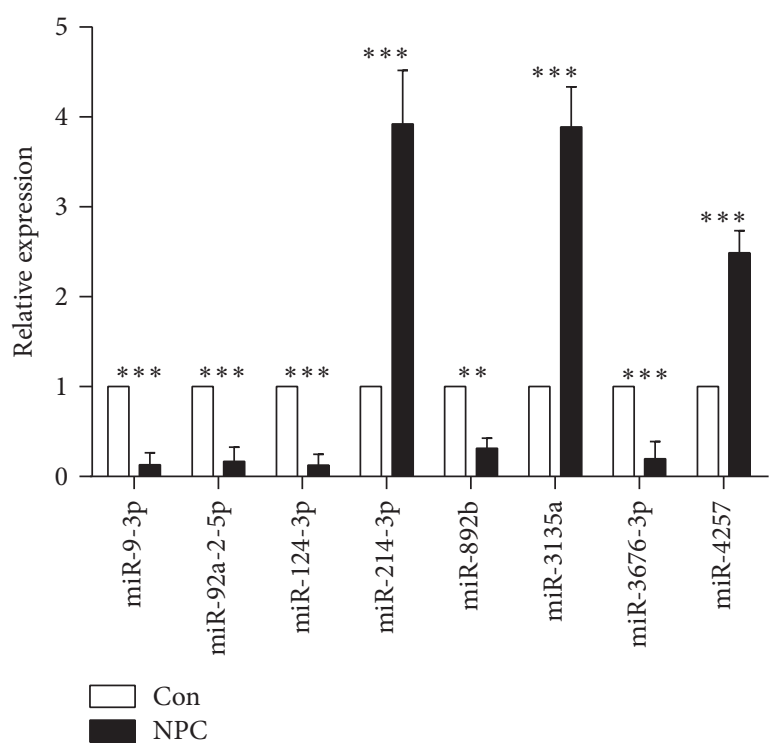

(b)

FIGURE 1: Selection and validation of plasma miRNA in NPC patients. (a) Heat map of differentially expressed microRNAs from GeneSpring software between the NPC group $(n=20)$ and the healthy control group $(n=10)$. Sixteen miRNAs were identified $(P<0.05)$. A vertical branch showed the expression pattern of candidate miRNAs in each individual. The relative expression was depicted according to the color scale shown in the figure. Red indicates upregulation and blue downregulation. Numbers with T indicate patients with NPC; numbers with $\mathrm{C}$ indicate healthy controls. (b) Validation of dysregulated miRNAs by qRT-PCR. An independent validation cohort included 102 patients with NPC and 35 healthy controls. Quantification was presented as mean values (error bars corresponded to standard deviation) relative to control from three independent experiments. ${ }^{* *} P<0.01$ and ${ }^{* * *} P<0.001$.

expressed plasma miRNAs, as identified by next-generation sequencing, could be helpful in predicting survival in patients with NPC [17]. All these studies suggested that circulating miRNA might serve as new biomarkers for NPC. However, the dynamic changes in these dysregulated miRNAs at different time points using self-paired plasma samples during the regular follow-up have never been explored.

Our previous study found that the level of plasma miR9 could distinguish locoregional from metastatic NPC cases with high sensitivity and specificity and was associated with clinical stages of NPC [19]. In this study, these eight plasma miRNAs screened from microarray were further identified by RT-qPCR in another follow-up cohort. All these eight plasma miRNAs were found to be differentially expressed between advanced stage and early stage of patients with NPC. The reason for this phenomenon was still not clear. Kosaka et al. suggested a secretary machinery of circulating miRNAs and their intercellular transfer and supposed that these circulating miRNAs might function as a pathway signal [22]. Further analysis is needed to clarify the origin of extracellular circulating miRNAs.

The diversification of circulating miRNA origin strongly suggests the existence of considerable interindividual differences in miRNA expression. Leidinger et al. monitored the plasma miRNA expression pattern in patients with lung cancer over time after surgery and found that the differences 

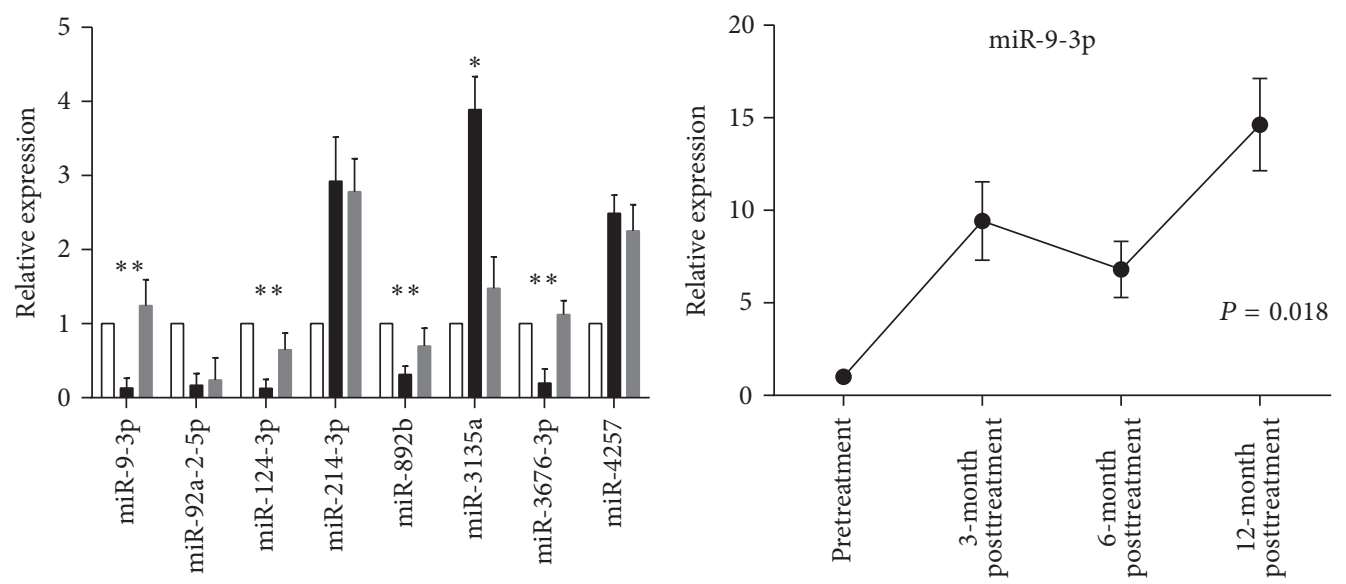

$\square$ Con

Pretreatment

3-month posttreatment

(a)

(b)
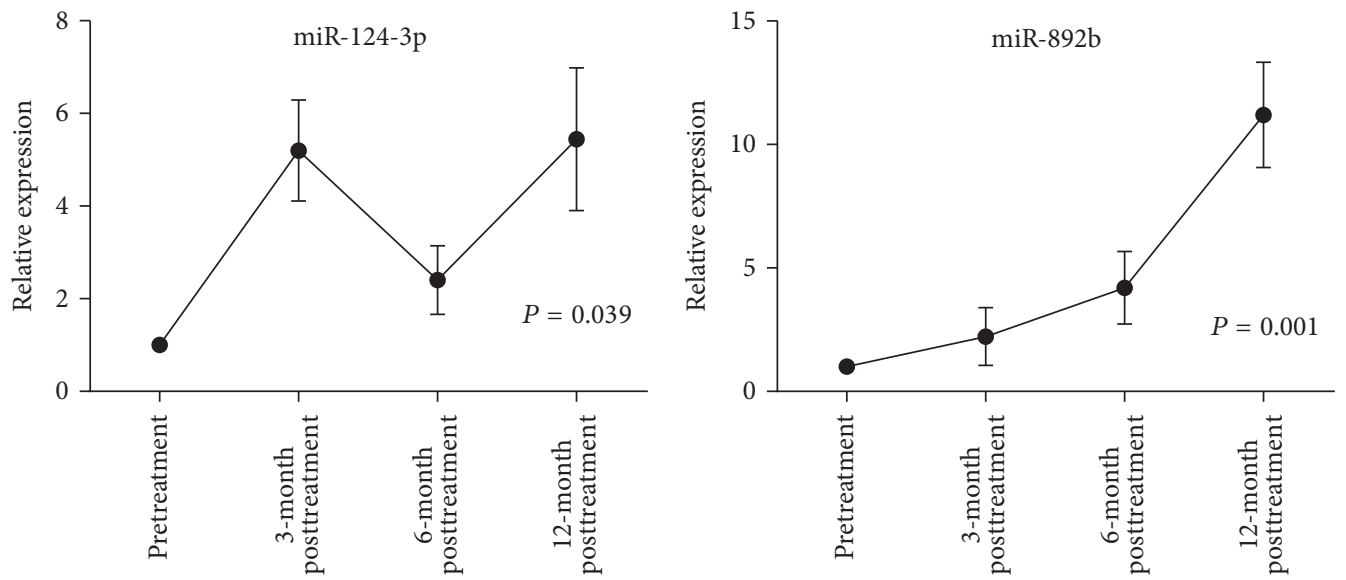

(c)

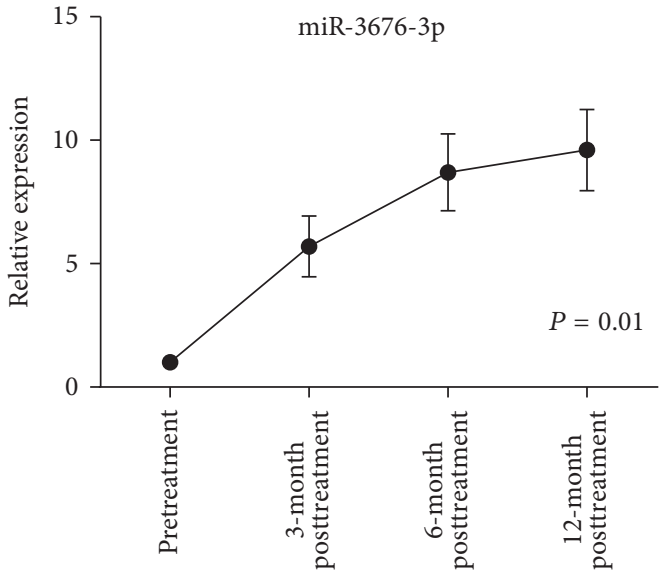

(d)

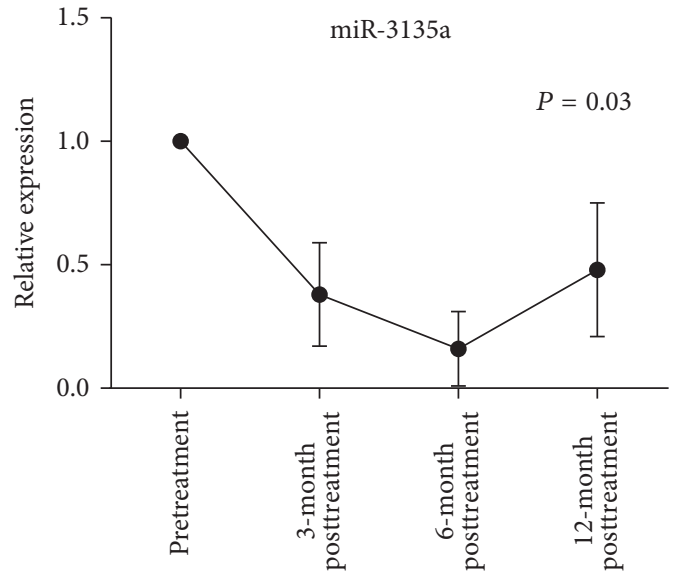

(e)

(f)

FIGURE 2: Dynamic changes in plasma miRNAs levels between pre- and posttreatment. (a) Levels of eight plasma miRNAs were examined in the paired samples of pretreatment and 3-month posttreatment of 90 patients compared with 35 healthy controls. The levels of five plasma miRNAs (miR-9-3p, miR-124-3p, miR-892b, miR-3135a, and miR-3676-3p) were significantly dysregulated and showed a tendency to go back to basic normal levels at 3-month posttreatment. (b-f) Dynamic changes in five plasma miRNA levels at three time points after treatment. Repeated-measure analysis of variance (ANOVA) was used to analyze the differences before treatment and 3, 6, and 12 months after treatment. ${ }^{*} P<0.05 ;{ }^{* *} P<0.01$. 


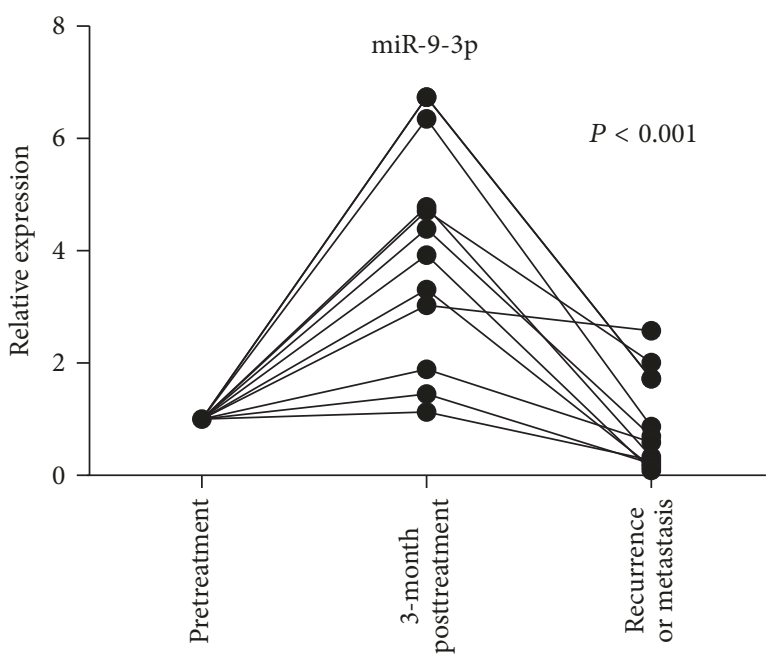

(a)

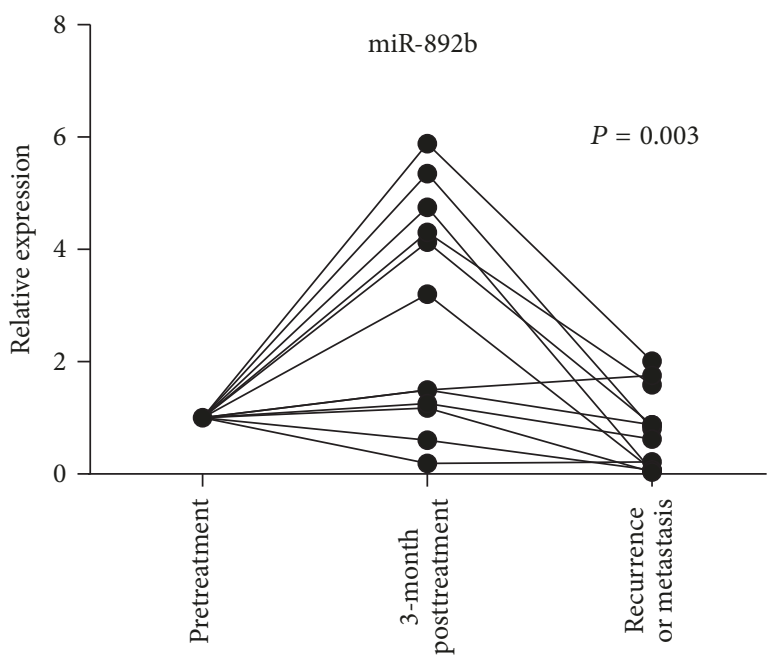

(c)

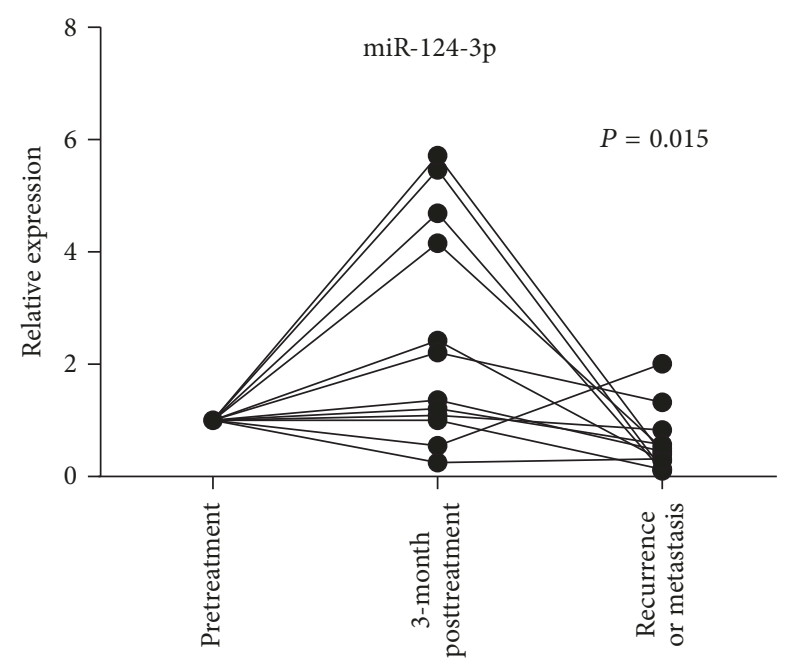

(b)

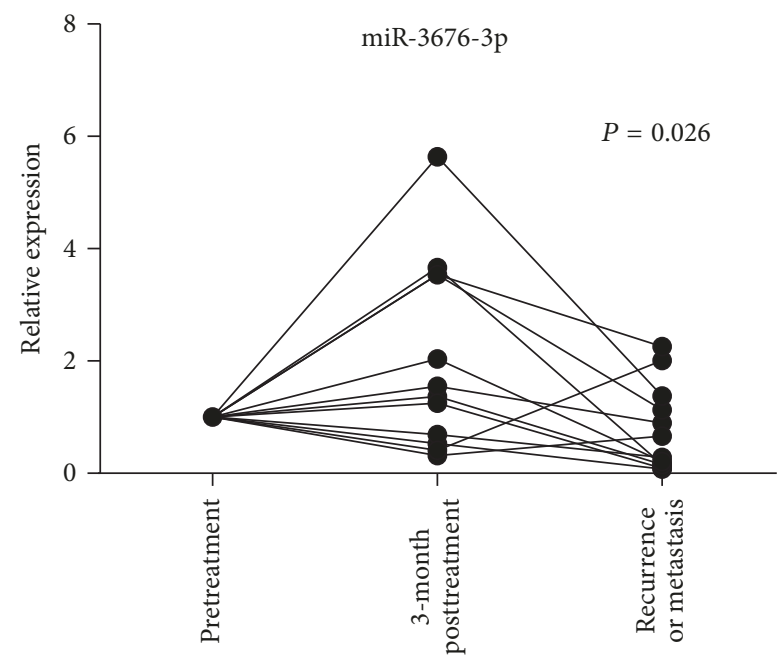

(d)

Figure 3: Dynamic changes in plasma miRNAs levels in NPC cases at the time of recurrence or metastasis. The levels of plasma miR-9$3 p$, miR-124-3p, miR-892b, and miR-3676-3p were statistically significantly upregulated at 3-month posttreatment and downregulated at recurrence or metastasis compared with pretreatment. Each individual value was represented by a circle, and the two points corresponding to the same individual were connected by a line.

in the overall miRNA patterns at different time points were significantly smaller than the differences between patients [23]. Paired plasma samples of pretreatment and posttreatment were collected in this study to eliminate the effects of interindividual differences on the results. Based on the results of microarray screening and qPCR validation, the study focused on 8 significantly dysregulated plasma miRNAs in the follow-up cohort including 102 patients with NPC and found that 5 plasma miRNAs (miR-9-3p, miR-124-3p, miR892b, miR-3135a, and miR-3676-3p) were significantly differentially expressed between pre- and posttreatment. Interestingly, the results showed that the levels of these five miRNAs tended to go back to basic normal levels after treatment compared with that before treatment, suggesting their potential values in monitoring recurrence and metastasis in patients with NPC.
Recent studies identified circulating miRNAs as predictors of response to therapeutics, such as radiotherapy and anticancer agents [24]. The abundance of circulating miRNAs was reported to change in patients with head and neck squamous cell carcinoma after radiochemotherapy [25]. Therefore, the dynamic changes in five plasma miRNA levels were further detected in the follow-up cohort. As shown in Figure 3, the levels of five plasma miRNAs tended to go back to basic normal levels after radiochemotherapy compared with pretreatment. The results suggested that these five miRNAs might have potential values in evaluating the therapeutic outcome of patients with NPC.

Circulating miRNAs have already been reported to predict the risk of recurrence in multiple cancers, including thyroid cancer [26], colon cancer [27], prostate cancer [28], and adrenocortical cancer [29]. In the long-term follow-up 
period, 12 cases had recurrence or metastasis at different time points after treatment. Also, the blood samples were collected, and the levels of the aforementioned five miRNAs were examined when the patient developed recurrence or metastasis. Surprisingly, the levels of four plasma miRNAs (miR-9-3p, miR-124-3p, miR-892b, and miR-3676-3p) were statistically significantly reduced at recurrence or metastasis. These results suggested that these four plasma miRNAs might serve as predictive biomarkers for monitoring recurrence and metastasis in patients with NPC. Although quantitative realtime PCR has been widely used to examine the expression of circulating miRNAs, still many arguments exist regarding the choice of the internal control. U6 and miR-16 were the most frequently used controls in recent studies on circulating miRNAs. However, U6 was reported to be absent in serum or plasma unless cell lysis occurred, and differential cell lysis was possible in diseases [30]. Additionally, hemolysis may cause up to 50-fold alteration of circulating miRNA levels [31]. In the present study, the dysregulated level of miR-16 was observed in the plasma of patients with NPC compared with healthy controls, whereas the levels of miR-634 and miR1228 were relatively stable in miRNA microarray profiling. Recently, miR-1228 was also proved to be a stable endogenous control for quantifying circulating microRNAs in cancer patients [32]. Therefore, the average level of U6, miR-634, and miR-1228 was used as an internal control.

Recent studies showed that the levels of circulating miR-9 and miR-124 were dysregulated, which might act as diagnostic and prognostic biomarkers in multiple cancers, including lung cancer, esophageal squamous cell carcinoma, pancreatic ductal adenocarcinoma, and prostate cancer [33-36]. In NPC, our previous studies showed that both miR-9 and miR-124 functioned as tumor suppressors by targeting chemokine receptor-4 (CXCR4) and forkhead box Q1 (FOXQ1), respectively $[37,38]$. It has also been reported that miR-9-3p suppresses the epithelial-mesenchymal transition (EMT) in NPC cells by downregulating fibronectin 1 (FN1), $\beta 1$ integrin (ITGB1), and $\alpha 5$ integrin (ITGAV) [39], while miR-124 suppresses tumor growth by targeting calpain small subunit 1 (CAPN4) and signal transducer and activator of transcription 3 (STAT3) [40, 41]. Although it has been reported that miR892b regulates the p19ARF/cyclin D1/CDK6 and Sp-1/MMP9 signaling networks in bladder cancer and miR-3676 regulates T-cell leukemia/lymphoma 1 (TCL1) in chronic lymphocytic leukemia $[42,43]$, the role of the three miRNAs (miR892b, miR-3135a, and miR-3676-3p) has never been reported in NPC. Further investigations are needed to reveal their functions and mechanisms in NPC tumorigenesis. Some of the NPC-related miRNAs found in previous studies were not identified in this study, including targeting of STAT3 by miRNA-98, targeting of TGF $\beta$ R2 by miR-93, and targeting of metastasis-associated gene 2 by miRNA-148b [44-46]. These partially inconsistent results may reflect differences in sample types, screening tools, or quantification methods. EBV-DNA and VCA-IgA have been tested routinely in NPC patients, because EBV infection is associated with the development of NPC. Positive EBV-DNA or VCA-IgA test might be a prognostic factor for the relapse and survival of NPC patients, but these markers do not always appear to be reliable [47].
We recognize that our study has limitations, including the fact that it was an observational study with limited sample size, and that the sensitivity and specificity of the miRNA biomarkers should be compared with conventional NPC markers such as EBV-DNA and VCA-IgA. Further prospective clinical trials with larger sample sizes, multicenter studies, and long-term follow-up should be performed to verify the specificity and sensitivity of these miRNA biomarkers.

In summary, this study clearly demonstrated that the dynamic changes in plasma miRNAs could be useful bloodbased biomarkers for predicting the therapeutic response of patients with NPC. These noninvasive blood-based biomarkers might have great potential in predicting the therapeutic response after radiochemotherapy and monitoring recurrence or metastasis. Therefore, our findings may suggest a new approach for the clinical follow-up of patients with NPC.

\section{Conflicts of Interest}

The authors declare that there are no conflicts of interest regarding the publication of this paper.

\section{Authors' Contributions}

Xia Xu, Juan Lu, and Fan Wang contributed equally to this work.

\section{Acknowledgments}

This work was partly funded by National Natural Science Foundation of China (U1132003 to Xiangping Li) and Natural Science Foundation of Guangdong Province, China (2014A030310032 to Xia Xu).

\section{References}

[1] M. L. K. Chua, J. T. S. Wee, E. P. Hui, and A. T. C. Chan, "Nasopharyngeal carcinoma," The Lancet, vol. 387, no. 10022, pp. 10121024, 2016.

[2] W. C. Cho, "Most common cancers in Asia-Pacific region: nasopharyngeal carcinoma," in Cancer Report of Asian-Pacific Region 2010, pp. 284-289, Asian Pacific Organization for Cancer Prevention, 2010.

[3] S. Qu, Z.-G. Liang, and X.-D. Zhu, "Advances and challenges in intensity-modulated radiotherapy for nasopharyngeal carcinoma," Asian Pacific Journal of Cancer Prevention, vol. 16, no. 5, pp. 1687-1692, 2015.

[4] A. D. King, A. C. Vlantis, T. W. C. Yuen et al., "Detection of nasopharyngeal carcinoma by MR imaging: Diagnostic accuracy of MRI compared with endoscopy and endoscopic biopsy based on long-term follow-up," American Journal of Neuroradiology, vol. 36, no. 12, pp. 2380-2385, 2015.

[5] N. Raab-Traub, "Nasopharyngeal carcinoma: An evolving role for the epstein-barr virus," Current Topics in Microbiology and Immunology, vol. 390, pp. 339-363, 2015.

[6] J.-F. Tang, Z.-H. Yu, T. Liu et al., "Five miRNAs as novel diagnostic biomarker candidates for primary nasopharyngeal carcinoma," Asian Pacific Journal of Cancer Prevention, vol. 15, no. 18, pp. 7575-7581, 2014. 
[7] W. Jiang, N. Liu, X.-Z. Chen et al., "Genome-wide identification of a methylation gene panel as a prognostic biomarker in nasopharyngeal carcinoma," Molecular Cancer Therapeutics, vol. 14, no. 12, pp. 2864-2873, 2015.

[8] G. A. Calin and C. M. Croce, "MicroRNA signatures in human cancers," Nature Reviews Cancer, vol. 6, no. 11, pp. 857-866, 2006.

[9] P. S. Mitchell, R. K. Parkin, E. M. Kroh et al., "Circulating microRNAs as stable blood-based markers for cancer detection," Proceedings of the National Acadamy of Sciences of the United States of America, vol. 105, no. 30, pp. 10513-10518, 2008.

[10] H. Schwarzenbach, D. S. B. Hoon, and K. Pantel, "Cell-free nucleic acids as biomarkers in cancer patients," Nature Reviews Cancer, vol. 11, no. 6, pp. 426-437, 2011.

[11] J. Zhang, H. Zhao, Y. Gao, and W. Zhang, "Secretory miRNAs as novel cancer biomarkers," Biochimica et Biophysica Acta (BBA) Reviews on Cancer, vol. 1826, no. 1, pp. 32-43, 2012.

[12] M. A. Cortez, C. Bueso-Ramos, J. Ferdin, G. Lopez-Berestein, A. K. Sood, and G. A. Calin, "MicroRNAs in body fluids-the mix of hormones and biomarkers," Nature Reviews Clinical Oncology, vol. 8, no. 8, pp. 467-477, 2011.

[13] H. Konishi, D. Ichikawa, S. Komatsu et al., "Detection of gastric cancer-associated microRNAs on microRNA microarray comparing pre- and post-operative plasma," British Journal of Cancer, vol. 106, no. 4, pp. 740-747, 2012.

[14] S. Zanutto, S. Pizzamiglio, M. Ghilotti et al., "Circulating miR378 in plasma: A reliable, haemolysis-independent biomarker for colorectal cancer," British Journal of Cancer, vol. 110, no. 4, pp. 1001-1007, 2014.

[15] X. Zeng, J. Xiang, M. Wu et al., "Circulating miR-17, miR-20a, miR-29c, and miR-223 combined as non-invasive biomarkers in nasopharyngeal carcinoma," PLoS ONE, vol. 7, no. 10, Article ID e46367, 2012.

[16] X. Liu, H.-N. Luo, W.-D. Tian et al., "Diagnostic and prognostic value of plasma microRNA deregulation in nasopharyngeal carcinoma," Cancer biology \& therapy, vol. 14, no. 12, pp. 11331142, 2013.

[17] N. Liu, R.-X. Cui, Y. Sun et al., "A four-miRNA signature identified from genome-wide serum miRNA profiling predicts survival in patients with nasopharyngeal carcinoma," International Journal of Cancer, vol. 134, no. 6, pp. 1359-1368, 2014.

[18] G. Zhang, J. Zong, S. Lin et al., "Circulating Epstein-Barr virus microRNAs miR-BART7 and miR-BART13 as biomarkers for nasopharyngeal carcinoma diagnosis and treatment," International Journal of Cancer, vol. 136, no. 5, pp. E301-E312, 2015.

[19] J. Lu, X. Xu, X. Liu et al., "Predictive value of miR-9 as a potential biomarker for nasopharyngeal carcinoma metastasis," British Journal of Cancer, vol. 110, no. 2, pp. 392-398, 2014.

[20] D. G. Pfister, K.-K. Ang, D. M. Brizel, R. S. Weber, G. T. Wolf, F. Worden et al., "Head and neck cancers," Journal of the National Comprehensive Cancer Network, vol. 9, pp. 596-650, 2011.

[21] K. J. Livak and T. D. Schmittgen, "Analysis of relative gene expression data using real-time quantitative PCR and the $2^{-\Delta \Delta C_{T}}$ method," Methods, vol. 25, no. 4, pp. 402-408, 2001.

[22] N. Kosaka, H. Iguchi, and T. Ochiya, "Circulating microRNA in body fluid: a new potential biomarker for cancer diagnosis and prognosis," Cancer Science, vol. 101, no. 10, pp. 2087-2092, 2010.

[23] P. Leidinger, A. Keller, C. Backes, H. Huwer, and E. Meese, "MicroRNA expression changes after lung cancer resection: A follow-up study," RNA Biology, vol. 9, no. 6, pp. 900-910, 2012.
[24] K. Zen and C.-Y. Zhang, "Circulating microRNAs: a novel class of biomarkers to diagnose and monitor human cancers," Medicinal Research Reviews, vol. 32, no. 2, pp. 326-348, 2012.

[25] I. Summerer, M. Niyazi, K. Unger et al., "Changes in circulating microRNAs after radiochemotherapy in head and neck cancer patients," Journal of Radiation Oncology, vol. 8, no. 1, article 296, 2013.

[26] J. C. Lee, J. T. Zhao, R. J. Clifton-Bligh et al., "MicroRNA-222 and MicroRNA-146b are tissue and circulating biomarkers of recurrent papillary thyroid cancer," Cancer, vol. 119, no. 24, pp. 4358-4365, 2013.

[27] N. Shivapurkar, L. M. Weiner, J. L. Marshall et al., "Recurrence of early stage colon cancer predicted by expression pattern of circulating microRNAs," PLoS ONE, vol. 9, no. 1, Article ID e84686, 2014.

[28] L. A. Selth, S. L. Townley, A. G. Bert et al., "Circulating microRNAs predict biochemical recurrence in prostate cancer patients," British Journal of Cancer, vol. 109, no. 3, pp. 641-650, 2013.

[29] O. Chabre, R. Libé, G. Assie et al., "Serum miR-483-5p and miR195 are predictive of recurrence risk in adrenocortical cancer patients," Endocrine-Related Cancer, vol. 20, no. 4, pp. 579-594, 2013.

[30] E. M. Kroh, R. K. Parkin, P. S. Mitchell, and M. Tewari, "Analysis of circulating microRNA biomarkers in plasma and serum using quantitative reverse transcription-PCR (qRT-PCR)," Methods, vol. 50, no. 4, pp. 298-301, 2010.

[31] C. C. Pritchard, E. Kroh, B. Wood et al., "Blood cell origin of circulating microRNAs: a cautionary note for cancer biomarker studies," Cancer Prevention Research, vol. 5, no. 3, pp. 492-497, 2012.

[32] J. Hu, Z. Wang, B.-Y. Liao et al., "Human miR-1228 as a stable endogenous control for the quantification of circulating microRNAs in cancer patients," International Journal of Cancer, vol. 135, no. 5, pp. 1187-1194, 2014.

[33] M. Sromek, M. Glogowski, M. Chechlinska et al., "Changes in plasma miR-9, miR-16, miR-205 and miR-486 levels after nonsmall cell lung cancer resection," Cellular Oncology, vol. 40, no. 5, pp. 529-536, 2017.

[34] Y. Cui, Y. Xue, S. Dong, and P. Zhang, "Plasma microRNA9 as a diagnostic and prognostic biomarker in patients with esophageal squamous cell carcinoma," Journal of International Medical Research, vol. 45, no. 4, pp. 1310-1317, 2017.

[35] B. Sun, X. Liu, Y. Gao, L. Li, and Z. Dong, "Downregulation of miR-124 predicts poor prognosis in pancreatic ductal adenocarcinoma patients," British Journal of Biomedical Science, vol. 73, no. 4, pp. 152-157, 2016.

[36] V. Medina-Villaamil, S. Martínez-Breijo, P. Portela-Pereira et al., "Circulating MicroRNAs in blood of patients with prostate cancer," Actas Urológicas Españolas (English Edition), vol. 38, no. 10, pp. 633-639, 2014.

[37] J. Lu, H. Luo, X. Liu et al., "miR-9 targets CXCR4 and functions as a potential tumor suppressor in nasopharyngeal carcinoma," Carcinogenesis, vol. 35, no. 3, Article ID bgt354, pp. 554-563, 2014.

[38] X. H. Peng, H. R. Huang, J. Lu et al., "MiR-124 suppresses tumor growth and metastasis by targeting Foxq1 in nasopharyngeal carcinoma," Molecular Cancer, vol. 13, p. 186, 2014.

[39] Y. Ding, Y. Pan, S. Liu, F. Jiang, and J. Jiao, "Elevation of MiR-9-3p suppresses the epithelial-mesenchymal transition of nasopharyngeal carcinoma cells via down-regulating FN1, 
ITGB1 and ITGAV," Cancer Biology \& Therapy, vol. 18, no. 6, pp. 414-424, 2017.

[40] H. Hu, G. Wang, and C. Li, "miR-124 suppresses proliferation and invasion of nasopharyngeal carcinoma cells through the Wnt/\&beta;-catenin signaling pathway by targeting Capn4," OncoTargets and Therapy, vol. 10, pp. 2711-2720, 2017.

[41] S. Xu, N. Zhao, L. Hui, M. Song, Z.-W. Miao, and X.-J. Jiang, "MicroRNA-124-3p inhibits the growth and metastasis of nasopharyngeal carcinoma cells by targeting STAT3," Oncology Reports, vol. 35, no. 3, pp. 1385-1394, 2016.

[42] S.-S. Shin, S.-S. Park, B. Hwang et al., "MicroRNA-892b influences proliferation, migration and invasion of bladder cancer cells by mediating the p19ARF/cyclin D1/CDK6 and Sp1/MMP-9 pathways," Oncology Reports, vol. 36, no. 4, pp. 23132320, 2016.

[43] V. Balatti, L. Rizzotto, C. Miller et al., “TCL1 targeting miR-3676 is codeleted with tumor protein p53 in chronic lymphocytic leukemia," Proceedings of the National Acadamy of Sciences of the United States of America, vol. 112, no. 7, pp. 2169-2174, 2015.

[44] J. Liu, W. Chen, Z. Chen et al., "The effects of microRNA-98 inhibits cell proliferation and invasion by targeting STAT3 in nasopharyngeal carcinoma," Biomedicine \& Pharmacotherapy, vol. 93, pp. 869-878, 2017.

[45] X. Lyu, W. Fang, L. Cai et al., "TGFbetaR2 is a major target of miR-93 in nasopharyngeal carcinoma aggressiveness," Molecular Cancer, vol. 13, no. 1, article 51, 2014.

[46] M. Wu, X. Ye, S. Wang, Q. Li, Y. Lai, and Y. Yi, "MicroRNA-148b suppresses proliferation, migration, and invasion of nasopharyngeal carcinoma cells by targeting metastasis-associated gene 2," OncoTargets and Therapy, vol. 10, pp. 2815-2822, 2017.

[47] F.-P. Zhao, X. Liu, Z.-M. Zhong et al., "Positivity of both plasma Epstein-Barr virus DNA and serum Epstein-Barr virus capsid specific immunoglobulin A is a better prognostic biomarker for nasopharyngeal carcinoma," BBA Clinical, vol. 2, pp. 88-93, 2014. 


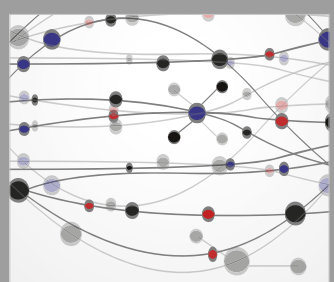

The Scientific World Journal
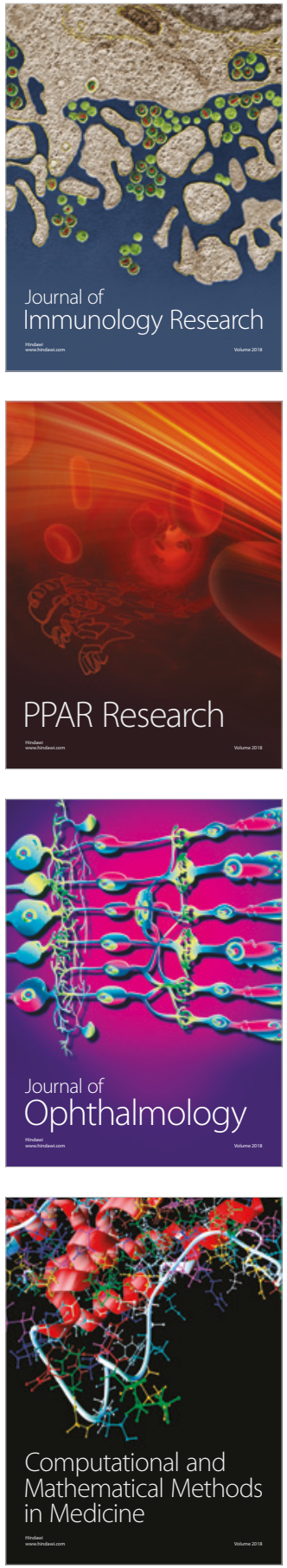

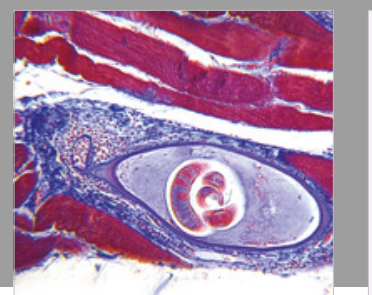

Gastroenterology Research and Practice

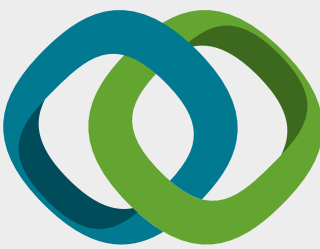

\section{Hindawi}

Submit your manuscripts at

www.hindawi.com
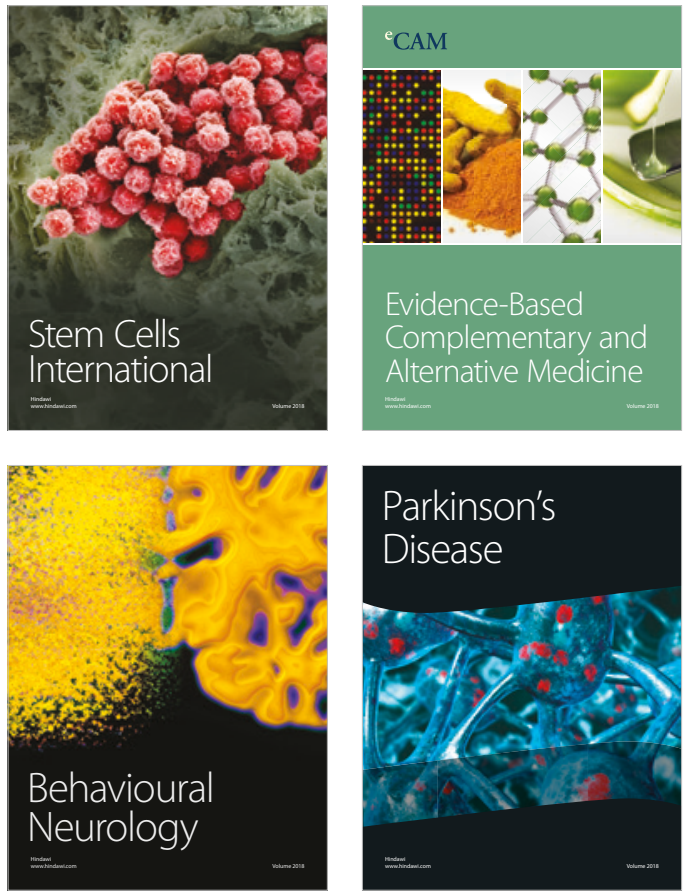

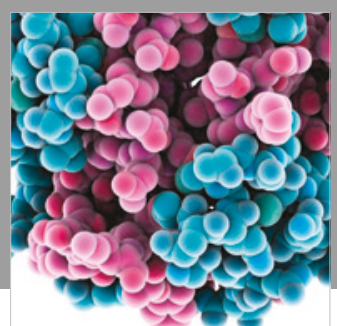

ournal of

Diabetes Research

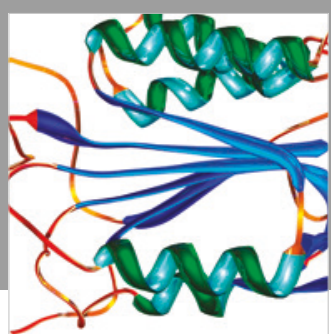

Disease Markers
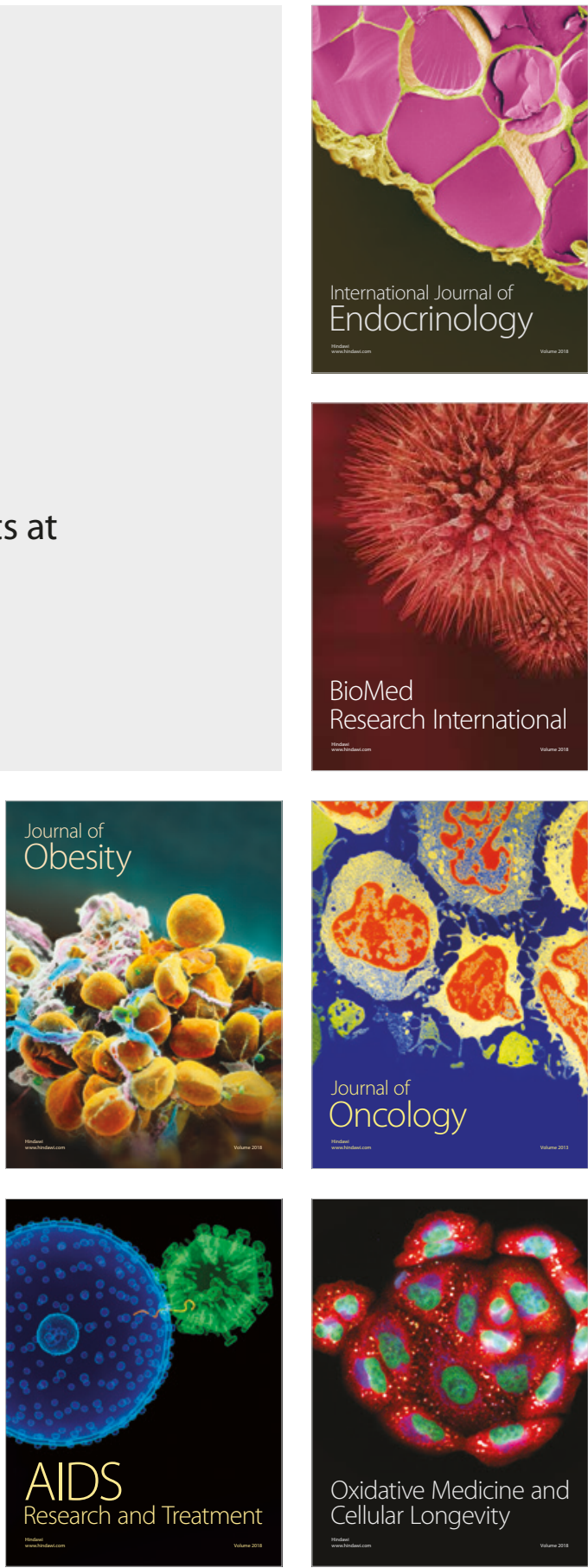